
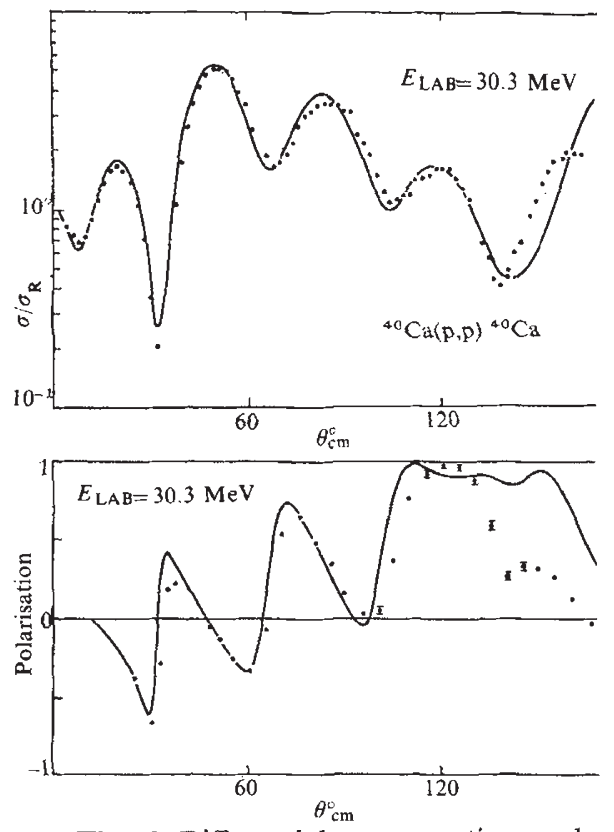

Fig. 2 Differential cross section and polarisation for the elastic scattering of $30.3 \mathrm{MeV}$ protons by ${ }^{40} \mathrm{Ca}$ compared with optical model calculations using the calculated potential.

tials with many adjustable parameters. In some respects they are even superior as they can give features of the cross sections that cannot be explained by phenomenological potentials.

This work is very promising, and needs to be extended and applied to a range of nuclei to enable its validity to be explored. It should also prove possible to extend the method to the calculation of the optical potentials appropriate to composite particles such as deuterons and alpha particles, and perhaps also to heavy ions. The availability of optical potentials that can be reliably calculated from fundamental data instead of being obtained by phenomenological analyses of elastic scattering data will be of the greatest value in nuclear structure studies.

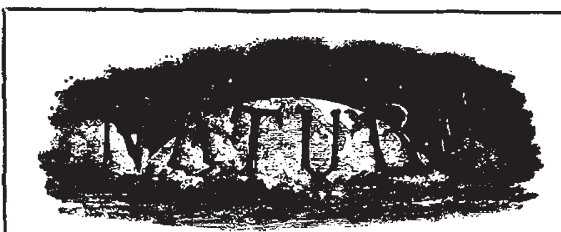

\section{A hundred years ago}

THE question has been discussed of late whether the ancient Greeks had an acute and true sense of colour. I remember once to have seen the remark that Sophocles shows his want of colour-sense by speaking of winecoloured ivy. Now this really shows how true his perception of colour was. I enclose two ivy leaves which I have gathered to-day off a wall; I could have gathered plenty of the same colour, which, as you see, is claret colour.

$$
\text { JOSEPH JOHN MURPHY }
$$

Old Forge, Dunmurry,

co. Antrim, October 21

From Nature 16, 25 October, 551; 1877.
Modulation of hormone receptors

from J. R. Tata

IT is now widely accepted that receptors for hormones and other cellular signals, whether located on the cell's surface or intracellular, turn over rapidly so that the modulation of receptor level can be an important element regulating a cell's activity. About two years ago an account of the first reports on the possibility that some hormones themselves may control the levels of their own receptors or receptors for other hormones appeared in these columns (News and Views 257, $741 ; 1975)$. Whereas autoregulation of hormonal receptors is still a matter of some controversy, many investigators now accept that one hormone can regulate the level of receptor for another. In some instances it is hard to see the physiological relevance of such interplay among hormones but a recent paper suggests that it may be of clinical significance.

Endocrinologists and cardiologists have for long been aware of the tachycardia accompanying hyperthyroidism or thyrotoxicosis and that cardiac function in such patients was abnormally sensitive to small changes in adrenaline levels. Williams et al. (J. biol. Chem. 252, 2787; 1977) now explain this wellknown syndrome by suggesting that thyroid hormones regulate the number of adrenergic receptor molecules in cardiac cells. In a carefully designed experiment they used the binding of the potent $\beta$-adrenergic antagonist ( - dihydroalprenolol, to compare the number and affinity of $\beta$-adrenergic receptors in membranes isolated from myocardium of normal rats and animals made hyperthyroid by injections of the two thyroid hormones, Lthyroxine and triiodo-L-thyronine. Within 3-7 days, this treatment caused an increase in the binding capacity of cardiac membranes from $89 \mathrm{fmol}$ of (-) - ${ }^{3} \mathrm{H}$-dihydroalprenolol per $\mathrm{mg}$ protein in normal (euthyroid) rats to 196 fmol per $\mathrm{mg}$ protein. On the other hand, there was no difference in the affinity of the receptor for dihydroalprenolol or the $\beta$-adrenergic agonist isoproterenol $\left(K_{0}\right.$ at equilibrium of 2-15 $\mathrm{nM}$ dihydroalprenolol) between membrane preparations from euthyroid and hyperthyroid animals. Williams et al. therefore conclude that thyroid hormones control the number but not the nature of $\beta$-adrenergic receptors in myocardial cells. This would explain the tachycardia and increased myocardial contractility in clinical hyperthyroidism. Their abservations would

J. R. Tata is at the National Institute of Medical Research, London. also explain an earlier report that cultured foetal mouse hearts were rendered more sensitive by triiodothyronine to the chronotropic effect of $\beta$-adrenergic receptor stimulation (Widenthal J. Pharmacol. exp. Therap. 190, $272 ; 1974)$. If the same were true of other cells, then these findings could also explain skeletal muscle tremor or the enhanced rate of glycogenolysis and lipolysis in hyperthyroid subjects or experimental animals.

Are the observations of Williams et al. merely a peculiarity of thyroid hormones or are they part of a general pattern of an interhormonal regulatory device? Several recent publications point to the latter possibility. Such evidence comes mainly from studies on the interaction between hormones regulating reproductive processes which are known to be under multiple hormonal control. To cite but a few examples, Charreau et al. (Molec. Cell Endocrinol. 7, 1; 1977) have put forward a novel explanation why androgen, which like other steroids is not thought to act on adenylate cyclase, causes an increase in cyclic AMP levels in the rat prostate. These workers found that dihydrotestosterone (the active form of testosterone in target cells) increases the number of receptors for prolactin, a hormone known to influence male sexual development and to have a marked effect on adenylate cyclase in other tissues such as the mammary gland. Any interpretation of long-term studies of this kind in whole animals suffers from the drawback that one may not be measuring direct effects of hormones on receptor levels. For this reason, the demonstration that FSH (follicle stimulating hormone) induced receptors for hCG (human chorionic gonadotropin) directly in vitro in rat granulosa cells (Nimrod et al. Nature $\mathbf{2 6 7}, 632 ; 1977$ ) is significant. Hormonal interplay can of course operate in both directions, namely that one hormone can also dampen the sensitivity of a cell to another. Thus, it is interesting to note a recent report by Bhakoo and Katzenellenbogen (Molec. Cell Endocrinol. 8, 121; 1977) in which progesterone is shown to suppress the synthesis of oestrogen receptor molecules in the uterus. The resulting suppression of the replenishment of receptor molecules would thus explain the lowered uterine sensitivity to oestrogen that is known to be provoked by progesterone.

It is not difficult to predict that we shall be seeing many more examples of hormonal modulation of hormone receptors, particularly in reproductive tissues, mammary gland and the neuroendocrine complex, where multiple hormones are involved in the regulation of their development or adult 